\title{
From lines to epipoles through planes in two views
}

\author{
C. Saguiés*, A.C. Murillo, F. Escudero, J.J. Guerrero \\ DIIS-I3A, Universidad de Zaragoza, C/ María de Luna num. 1, E-50018 Zaragoza, Spain
}

Received 28 June 2004; received in revised form 16 March 2005; accepted 15 June 2005

\begin{abstract}
This paper addresses the computation of the fundamental matrix between two views, when camera motion and 3D structure are unknown, but planar surfaces can be assumed. We use line features which are automatically matched in two steps. Firstly, with image based parameters, a set of matches are obtained to secondly compute homographies, which allows to reject wrong ones, and to grow good matches in a final stage. The inclusion of projective transformations gives much better results to match features with short computing overload. When two or more planes are observed, different homographies can be computed, segmenting simultaneously the corresponding planar surfaces. These can be used to obtain the fundamental matrix, which gives constraints for the whole scene. The results show that the global process is robust enough, turning out stable and useful to obtain matches and epipolar geometry from lines in man made environments.
\end{abstract}

(C) 2005 Pattern Recognition Society. Published by Elsevier Ltd. All rights reserved.

Keywords: Robust line matching; Multi-plane scenes; Homographies; Epipolar geometry; Fundamental matrix

\section{Introduction}

The fundamental matrix encapsulates the geometric information which relates two different views regardless of the observed scene. The non-metric basis of this matrix makes possible to use uncalibrated cameras. It has been normally computed using points [1] which fails when all the points lie in a plane or with small baseline images. Besides, it cannot be computed directly from lines. It can be obtained from lines, however, if they are in different planes through the corresponding homographies [2]. The use of lines instead of points has advantages, mainly in man made environments, because the lines can be accurately extracted in noisy images, they capture more information than points and they may be matched where partial occlusions occur [3].

In this context the matching problem is crucial to make the process work automatically. The matching of features based on image parameters has many drawbacks, giving non-matched or wrong matched features. Projective

\footnotetext{
This work was supported by project DPI2003-07986.

* Corresponding author. Tel.: +34976761940; fax: 34976761914

E-mail address: csagues@unizar.es (C. Sagüés).
}

transformations allow image dependent measures, as crosscorrelation, to be a view-point invariant which makes possible to afford wide baseline matching [4]. So, the epipolar constraint imposed by the fundamental matrix can be used for the matching of points, but it cannot be used for line matching, because the end points of the extracted lines are not reliable. If the features lie on a planar surface then a plane projective transformation can be used.

Scenes with several planes are usual in man made environments, and the model to work with multiple views of them is well known. Points or lines in one image of the world plane are mapped to points or lines in the other image by a plane to plane homography, also known as a plane projective transformation [5]. This is an exact transformation for planar scenes or for null baseline image pairs. We robustly match lines between two images using the projective transformations corresponding to the existing scene planes [6]. The robust line matching and the computation of the corresponding homography is iteratively carried out until we have no more available planes. If two planes have been computed at least, the fundamental matrix can be computed, which gives a general constraint for the whole scene. It has been reported that the multi-plane algorithm is unstable, but when only two planes are observed, which is quite usual in man 
made environments, the multi-plane algorithm gives better results than the general method [7].

In this paper the automatic matching of lines computing homographies and its use to obtain the epipoles in an image pair is exposed and discussed. Some results related to the accuracy of the computed fundamental matrix, the segmentation of planes and the simultaneous matching of lines are given. To get enough initial basic matches, scenes where two planes take up most part of the images are required.

After this introduction, we present in Section 2 the matching of lines based in the simultaneous estimation of homographies. After that, we present in Section 3 the equations which relate the homographies and the fundamental matrix. The conditioning of the motion and the scene for the fundamental matrix to be computed is exposed in Section 4. Experimental results with real images and synthetic data are presented in Section 5. Finally, Section 6 is devoted to expose the conclusions. The availability of both, homographies and the fundamental matrix may be useful in other applications. For example, we have used it for robot homing [8].

\section{Robust matching}

We take straight lines as key features because, as said before, they are plentiful in man made environments, they have a simple mathematical representation, they can be extracted more accurately than points and they behave better with partial occlusions.

\subsection{Basic matches}

In the first stage we match the lines using image information. We take into account not only the geometric parameters of the lines, but also the brightness attributes supplied by the line extractor. So, average grey level $(a g l)$ and contrast $(c)$, are combined with geometric parameters of the segments, such as midpoint coordinates $\left(x_{m}, y_{m}\right)$, line orientation $(\theta$ in $2 \pi$ range with dark on the right and bright on the left) and its length $(l)$.

The lines are stated as compatible when they have geometric and brightness similarity. The match between two lines is made to the weighted nearest neighbor. So, naming $\mathbf{r}_{\mathbf{g}}$ the difference of geometric parameters between both images $(1,2), \mathbf{r}_{\mathbf{g}}=\left[x_{m 1}-x_{m 2}, y_{m 1}-y_{m 2}, \theta_{1}-\theta_{2}, l_{1}-l_{2}\right]^{\mathrm{T}}$ and $\mathbf{r}_{\mathbf{b}}$ the variation of the brightness parameters between both images, $\mathbf{r}_{\mathbf{b}}=\left[a g l_{1}-a g l_{2}, c_{1}-c_{2}\right]^{\mathrm{T}}$, we can compute two Mahalanobis distances, one for the geometric parameters $\mathbf{d}_{\mathbf{g}}=\mathbf{r}_{\mathbf{g}}^{\mathrm{T}} \mathbf{S}^{-1} \mathbf{r}_{\mathbf{g}}$ and other for the brightness parameters, $\mathbf{d}_{\mathbf{b}}=\mathbf{r}_{\mathbf{b}}^{\mathrm{T}} \mathbf{B}^{-1} \mathbf{r}_{\mathbf{b}}$. Here, $\mathbf{S}$ is a matrix that expresses the uncertainty due to extraction noise and the uncertainty due to camera motion and unknown scene structure, and $\mathbf{B}$ is a matrix that expresses the uncertainty due to measurement noise and changes of illumination. From these distances, the similarity is measured by the simultaneous compliance of geometric and brightness compatibility tests,
- Geometric compatibility. Assuming that the noise is Gaussian distributed, the similarity distance for the geometric parameters is distributed as a $\chi^{2}$ with 4 d.o.f. Establishing a significance level of 5\%, the compatible lines must fulfill, $\mathbf{d}_{\mathbf{g}} \leqslant \chi_{4}^{2}(95 \%)$.

- Brightness compatibility. Similarly, referring to the brightness parameters, the compatible lines must fulfill, $\mathbf{d}_{\mathbf{b}} \leqslant \chi_{2}^{2}(95 \%)$.

\subsection{Matches and homographies}

From matched lines $\left(\mathbf{n}_{1}, \mathbf{n}_{2}\right)$ belonging to the same plane, a projective transformation $\mathbf{H}_{21}$ exits, in such a way that $\mathbf{n}_{2}=\left[\mathbf{H}_{21}^{-1}\right]^{\mathrm{T}} \mathbf{n}_{1}$, being $\mathbf{H}_{21}$ the $3 \times 3$ projective transformation of points $\mathbf{x}_{2}=\mathbf{H}_{21} \mathbf{x}_{1}$.

We obtain the projective transformation of points, but using matched lines. To deduce it, we suppose the start $(s)$ and end (e) tips of a couple of matched lines to be $\mathbf{x}_{s 1}, \mathbf{x}_{e 1}, \mathbf{x}_{s 2}, \mathbf{x}_{e 2}$, which usually will not be corresponding points. As the tips belong to the line, we have that $\mathbf{x}_{s 2}^{\mathrm{T}} \mathbf{n}_{2}=0$; $\mathbf{x}_{e 2}^{\mathrm{T}} \mathbf{n}_{2}=0$. Besides, the tips of line in the first image, once transformed, also belong to the corresponding line in the second image, and therefore, we can write, $\mathbf{x}_{s 1}^{\mathrm{T}} \mathbf{H}_{21}^{\mathrm{T}} \mathbf{n}_{2}=0$; $\mathbf{x}_{e 1}^{\mathrm{T}} \mathbf{H}_{21}^{\mathrm{T}} \mathbf{n}_{2}=0$. Combining both equations, we have

$\mathbf{x}_{s 1}^{\mathrm{T}} \mathbf{H}_{21}^{\mathrm{T}}\left[\mathbf{x}_{s 2}\right]_{\times} \mathbf{x}_{e 2}=0 ; \quad \mathbf{x}_{e 1}^{\mathrm{T}} \mathbf{H}_{21}^{\mathrm{T}}\left[\mathbf{x}_{s 2}\right]_{\times} \mathbf{x}_{e 2}=0$,

where $\left[\mathbf{x}_{S 2}\right]_{\times}$is the skew-symmetric matrix obtained from the vector $\mathbf{x}_{s 2}$.

Therefore, each couple of corresponding lines gives two homogeneous equations to compute the projective transformation, which can be determined up to a non-zero scale factor. To compute the homography, we have chosen the ransac algorithm [9], which is a robust method to consider the existence of outliers. It makes a search in the space of solutions obtained from subsets of four matches. Each subset provides a $8 \times 9$ system of equations whose solution is obtained from singular value decomposition.

From here on, we introduce the geometrical constraint introduced by the estimated homography to get a bigger set of matches. Our objective is to obtain at the end of the process more good matches, and to discard the wrong ones obtained in the first stage, which was based on image properties. Thus, final matches are composed by two sets. The first one is obtained from the matches selected after the robust computation of the homography that passes additionally an overlapping test compatible with the transformation of the segment tips. The second set of matches is obtained taking all the segments not matched initially and those being rejected previously. With these lines, a matching process similar to the basic matching is carried out. However, now the matching is made to the nearest neighbor segment transformed with the homography. The transformation is applied to the end tips of the image segments using the homography $\mathbf{H}_{21}$ to find, not only compatible lines, but also compatible segments in the same line. In the first stage of the matching process there 


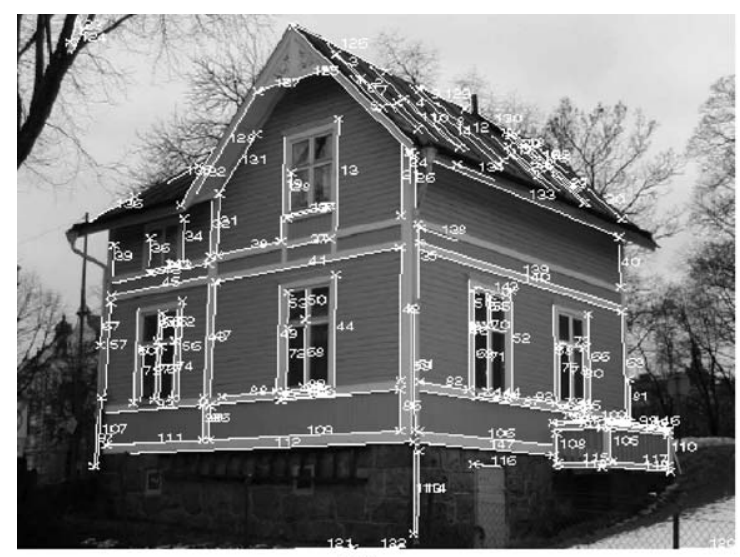

(a)

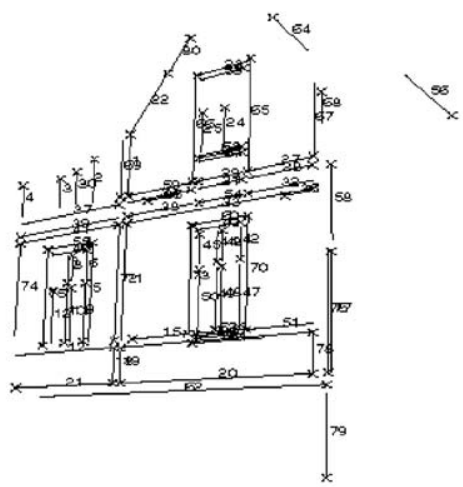

(c)

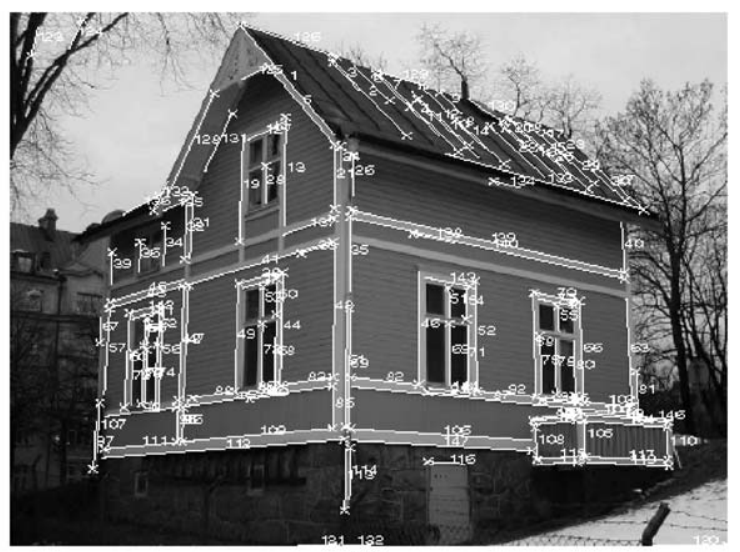

(b)

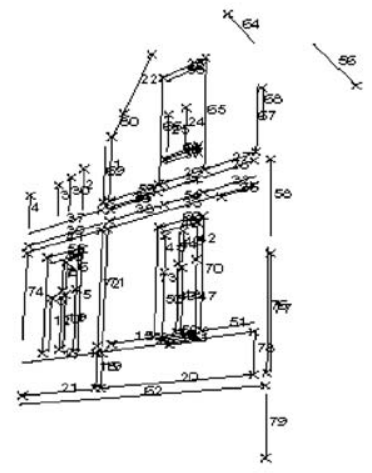

(d)
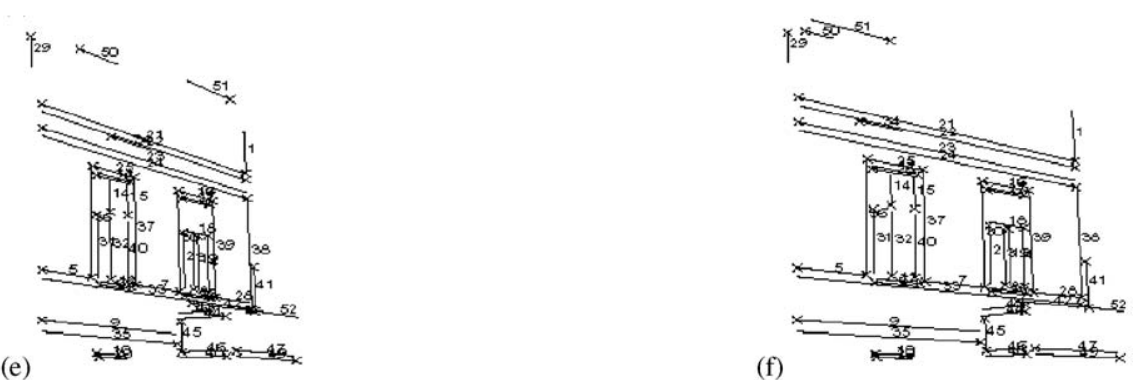

Fig. 1. House: Image pair used to compute the homographies and the epipole. (a), (b) Basic matches. (c), (d) Matches corresponding to the first homography. (e), (f) Matches corresponding to the second one. (Original images from KTH, Stockholm).

was no previous knowing of camera motion. However, in this second step, the computed homography provides information about expected disparity due to motion and therefore the uncertainty of geometric variations can be reduced.

Details of this method of matching and the experiments introducing the goodness of the projective transformation to obtain better matches have been presented previously [6].

\section{From homographies to the fundamental matrix}

The fundamental matrix has been stated as a crucial tool when using uncalibrated images. As known, it is a $3 \times 3$ matrix of rank 2 which encapsulates the epipolar geometry, and it only depends on internal parameters of the camera and on the relative motion.
Let us suppose the images are obtained with the same camera whose projection matrixes in a common reference system are $\mathbf{P}_{1}=\mathbf{K}[\mathbf{I} \mid \mathbf{0}], \mathbf{P}_{2}=\mathbf{K}[\mathbf{R} \mid \mathbf{t}]$ (being $\mathbf{R}$ the camera rotation, $\mathbf{t}$ the translation and $\mathbf{K}$ the internal calibration matrix). Then, the fundamental matrix can be expressed as $\mathbf{F}_{21}=\mathbf{K}^{-\mathrm{T}}\left([\mathbf{t}]_{\times} \mathbf{R}\right) \mathbf{K}^{-1}$. Normally, it has been computed from corresponding points $[1,10,11]$, using the epipolar constraint, which can be expressed as $\mathbf{x}_{2}^{\mathrm{T}} \mathbf{F}_{21} \mathbf{x}_{1}=0$. However, the fundamental matrix is unstable when points lie close to planes [7].

Lines cannot be used to compute the fundamental matrix directly, but they can be used to compute it through homographies, in such a way that: $\mathbf{F}_{21}=\left[\mathbf{e}_{2}\right]_{\times} \mathbf{H}_{21}^{\pi}$, where $\mathbf{e}_{2}$ is the epipole of the second image and $\mathbf{H}_{21}^{\pi}$ is the homography between first and second image through plane $\pi$. If at 


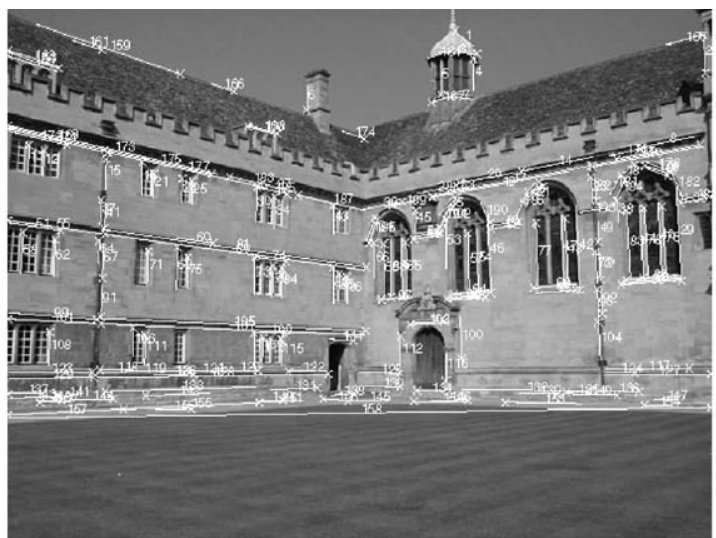

(a)

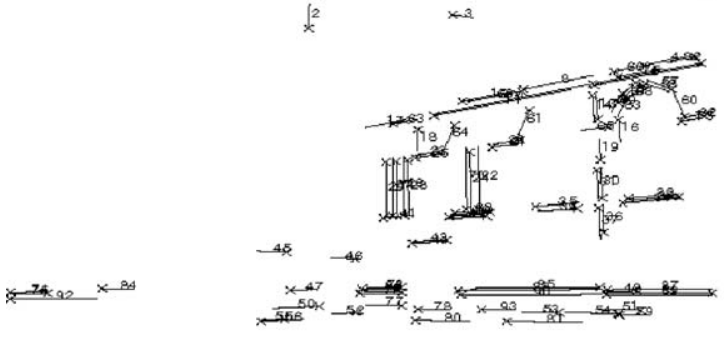

(c)

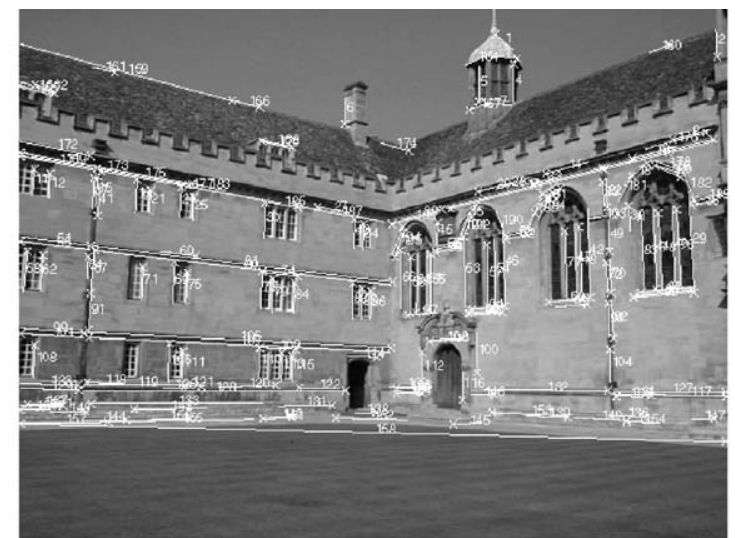

(b)

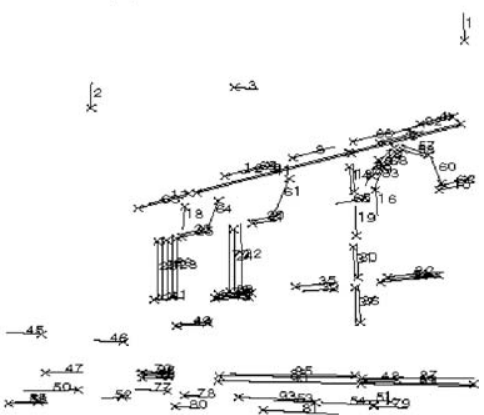

(d)
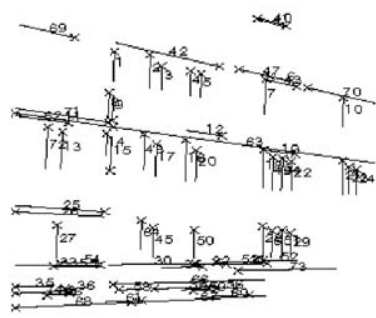

(e)

(f)

Fig. 2. College: Image pair used to compute the homographies and the epipole. (a), (b) Basic matches. (c), (d) Matches corresponding to the first homography. (e), (f) Matches corresponding to the second one. (Original images from VGG, Oxford).

least two homographies $\left(\mathbf{H}_{21}^{\pi_{1}}, \mathbf{H}_{21}^{\pi_{2}}\right)$ can be computed between both images corresponding to two planes, a homology $\mathbf{H}=\mathbf{H}_{21}^{\pi_{1}} \cdot\left(\mathbf{H}_{21}^{\pi_{2}}\right)^{-1}$, that is a mapping from one image into itself, exists. Under this mapping the epipole is a fixed point $\mathbf{e}_{2}=\mathbf{H} \mathbf{e}_{2}$, so it may be determined from the eigenvector of $\mathbf{H}$ corresponding to non-unary eigenvalue [5]. Thus, the fundamental matrix can be computed with either of the following as,

$$
\begin{aligned}
& \mathbf{F}_{21}=\left[\mathbf{e}_{2}\right]_{\times} \mathbf{H}_{21}^{\pi_{1}}, \\
& \mathbf{F}_{21}=\left[\mathbf{e}_{2}\right]_{\times} \mathbf{H}_{21}^{\pi_{2}},
\end{aligned}
$$

being $\left[\mathbf{e}_{2}\right]_{\times}$the skew matrix corresponding to $\mathbf{e}_{2}$ vector.

Alternatively, the fundamental matrix can be computed from homographies using directly the epipolar constraint $\mathbf{x}_{2}^{\mathrm{T}} \mathbf{F}_{21} \mathbf{x}_{1}=0$. We have two homographies obtained for planes
Table 1

Matches of scenes in Fig. 1 (House) and Fig. 2 (College)

\begin{tabular}{lllll}
\hline & Basic matches & Good & Final matches & Good \\
\hline House & 148 & $75 \%$ & $114.6(8.91)$ & $99 \%(1 \%)$ \\
College & 196 & $82 \%$ & $156.7(11.09)$ & $96 \%(1 \%)$ \\
\hline
\end{tabular}

Only the lines on the two main planes are considered. We have repeated the experiments one hundred times using the same Basic matches and we show as Final matches the mean values and, in brackets, the standard deviation.

( $\pi_{1}$ and $\pi_{2}$ ) which relate the coordinates of the points of the planes in both images, in such a way that

$$
\begin{aligned}
& \mathbf{x}_{2}^{\mathrm{T}} \mathbf{F}_{21} \mathbf{x}_{1}=\left(\mathbf{H}_{21}^{\pi_{1}} \mathbf{x}_{1}\right)^{\mathrm{T}} \mathbf{F}_{21} \mathbf{x}_{1}=\mathbf{x}_{1}^{\mathrm{T}} \mathbf{H}_{21}^{\pi_{1} \mathrm{~T}} \mathbf{F}_{21} \mathbf{x}_{1}=0, \\
& \mathbf{x}_{2}^{\mathrm{T}} \mathbf{F}_{21} \mathbf{x}_{1}=\left(\mathbf{H}_{21}^{\pi_{2}} \mathbf{x}_{1}\right)^{\mathrm{T}} \mathbf{F}_{21} \mathbf{x}_{1}=\mathbf{x}_{1}^{\mathrm{T}} \mathbf{H}_{21}^{\pi_{2} \mathrm{~T}} \mathbf{F}_{21} \mathbf{x}_{1}=0 .
\end{aligned}
$$



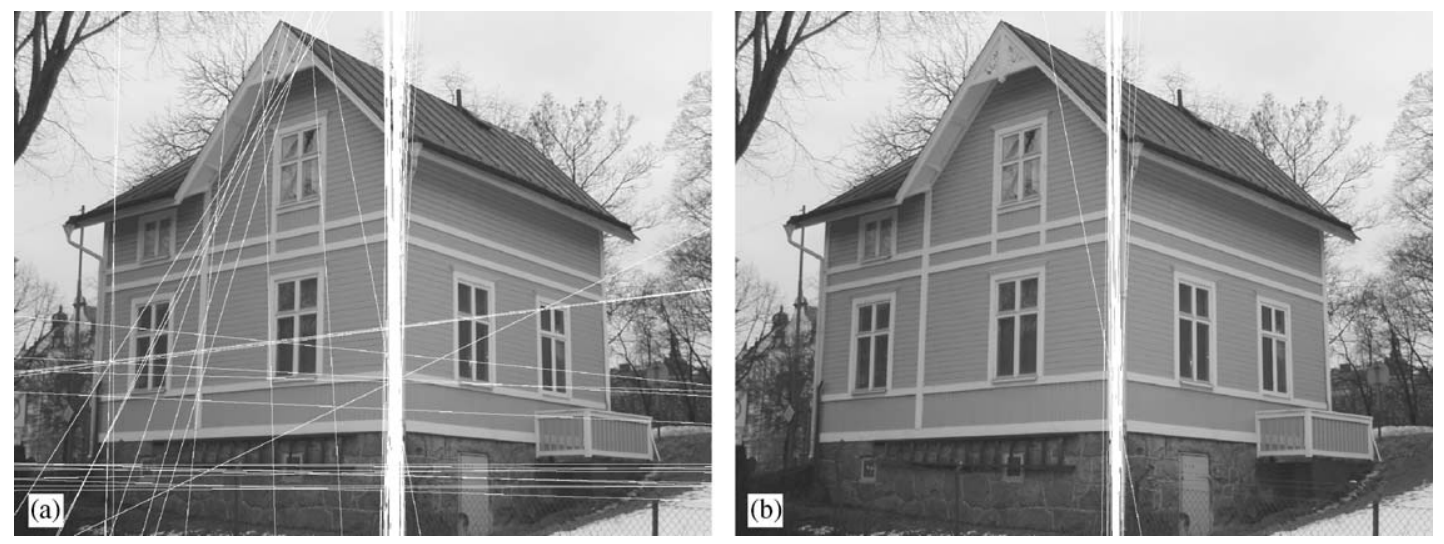

Fig. 3. Intersection lines of two planes through the eigenvalues of the homology. The lines corresponding to 100 runs are represented without the homology filter (a) and with it (b).
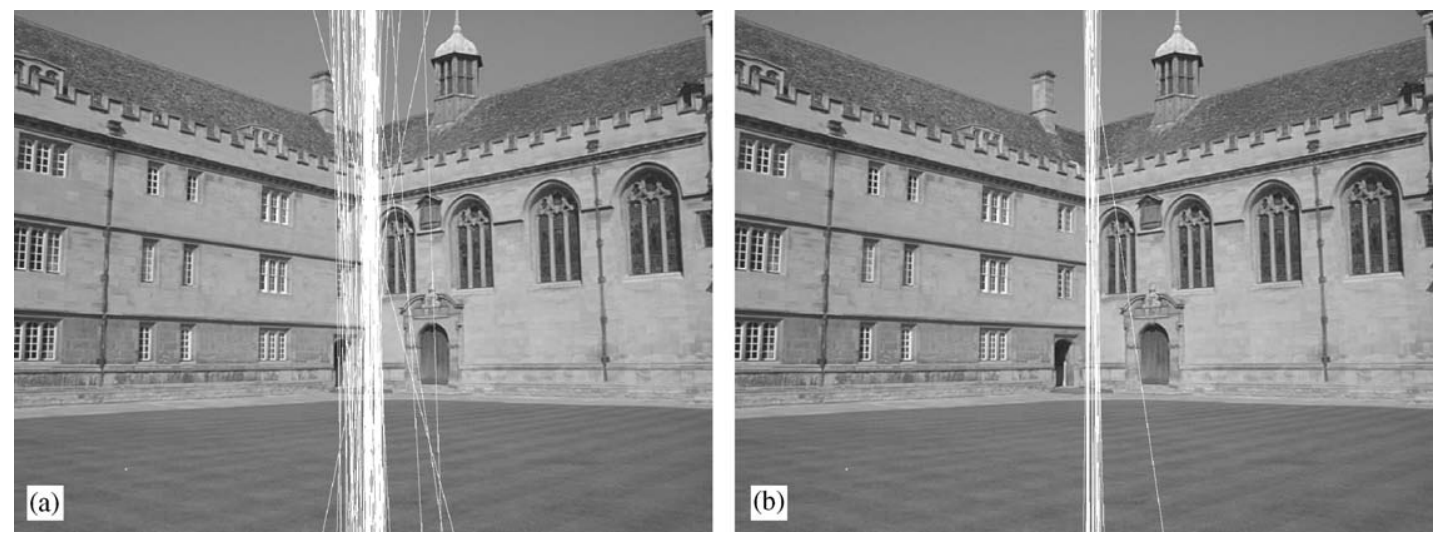

Fig. 4. Intersection lines of two planes through the eigenvalues of the homology. The lines corresponding to 100 runs are represented without the homology filter (a) and with it (b).

As this relation is satisfied by any point $\mathbf{x}_{1}$, we can write the following relations [7]:

$\mathbf{H}_{21}^{\pi_{1} \mathrm{~T}} \mathbf{F}_{21}+\mathbf{F}_{21}^{\mathrm{T}} \mathbf{H}_{21}^{\pi_{1}}=0$

$\mathbf{H}_{21}^{\pi_{2} \mathrm{~T}} \mathbf{F}_{21}+\mathbf{F}_{21}^{\mathrm{T}} \mathbf{H}_{21}^{\pi_{2}}=0$.

From here, a system of twelve linear equations with the elements of the fundamental matrix is available and it can be solved using singular value decomposition.

\section{Conditioning of scene and motion on the fundamental matrix}

As known, the goodness of the fundamental matrix depends on motion, in such a way that if the two camera centers are coincident, then the epipolar geometry is not defined. Similarly, when points or lines of the images are in a single scene plane, the fundamental matrix cannot be computed. As we propose to compute the fundamental matrix from homographies, a check on the homology conditioning may help to determine if the fundamental matrix may or may not be computed.

As said, $\mathbf{H}=\left(\mathbf{H}_{21}^{\pi_{1}}\right)^{-1} \cdot \mathbf{H}_{21}^{\pi_{2}}$ and taking into account that for a plane $\mathbf{H}_{21}=\mathbf{K}\left(\mathbf{R}-\left(\mathbf{t n}_{\pi}^{\mathrm{T}} / d_{\pi}\right)\right) \mathbf{K}^{-\mathbf{1}}$, it turns out that

$\mathbf{H}=\left(\mathbf{K}\left(\mathbf{R}-\frac{\mathbf{t n}_{\pi_{1}}^{\mathrm{T}}}{d_{\pi_{1}}}\right) \mathbf{K}^{-1}\right)^{-1}\left(\mathbf{K}\left(\mathbf{R}-\frac{\mathbf{t n}_{\pi_{\mathbf{2}}}^{\mathrm{T}}}{d_{\pi_{2}}}\right) \mathbf{K}^{-\mathbf{1}}\right)$,

being $\mathbf{n}_{\pi_{1}}, \mathbf{n}_{\pi_{2}}$ the normals and $d_{\pi_{1}}, d_{\pi_{2}}$ the distances to the planes.

Using the Sherman-Morrison formula [12], in a similar way than in Ref. [13], it turns out that

$$
\left(\mathbf{R}-\frac{\mathbf{t}_{\pi_{1}}^{\mathrm{T}}}{d_{\pi_{1}}}\right)^{-1}=\mathbf{R}^{-1}+\frac{\left(\mathbf{R}^{-1} \mathbf{t}\right)\left(\left(\mathbf{n}_{\pi_{1}}^{\mathrm{T}} / d_{\pi_{1}}\right) \mathbf{R}^{-1}\right)}{I+\mathbf{n}_{\pi_{1}}^{\mathrm{T}} \mathbf{R}^{-1} \mathbf{t}} .
$$

Substituting in Eq. (6) and operating, we obtain

$\mathbf{H}=\mathbf{I}+\mathbf{v p}^{\mathrm{T}}$, 

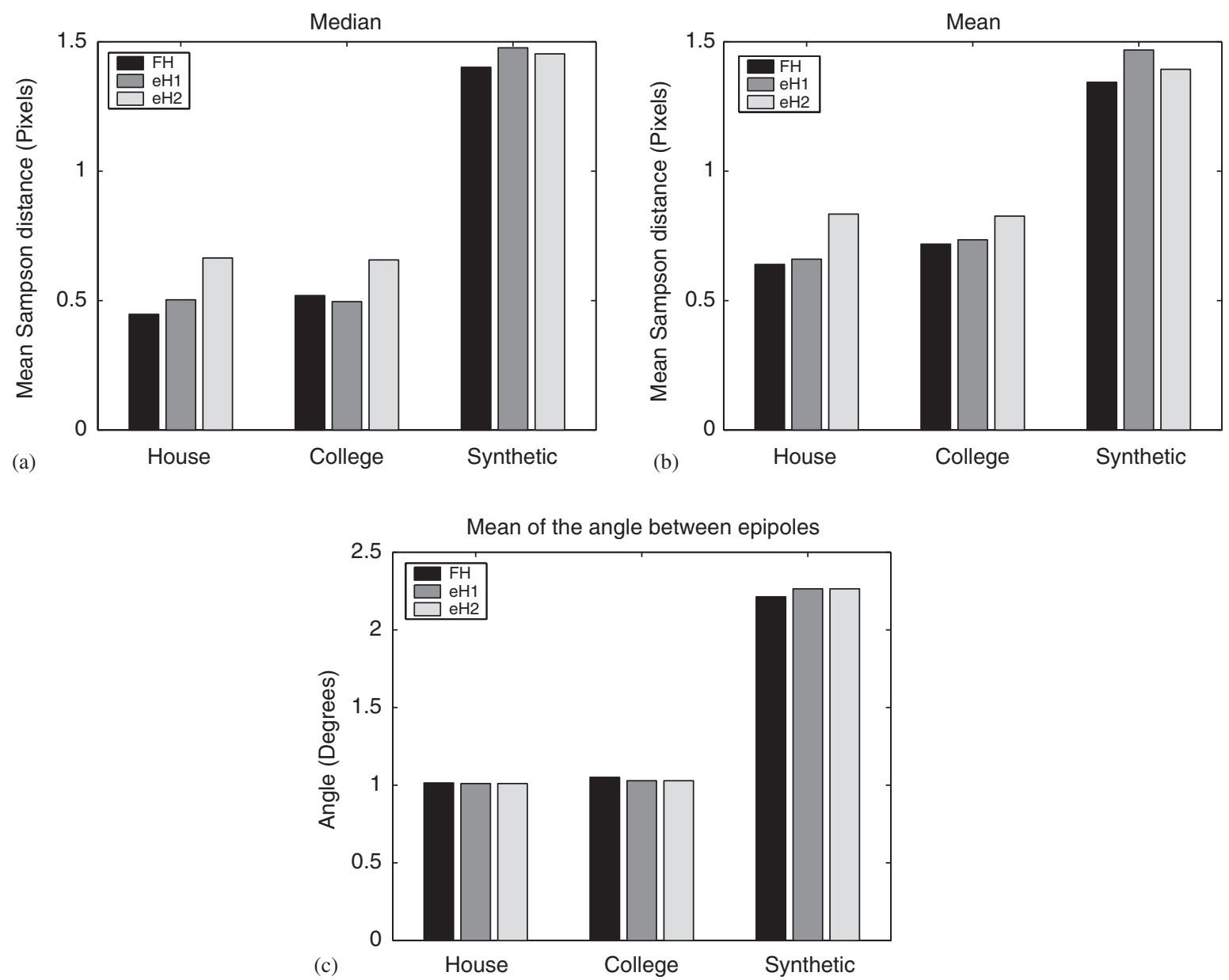

Fig. 5. Mean Sampson distance for 20 test points manually matched in the images of the House, the College and Synthetic. Median (a) and mean (b), in 100 executions. Mean of the angle with the three image pairs, in 100 executions, between the theoretical epipole and the computed one (c). These results are shown for the three ways of computing F: FH using Eq. (5), eH1 using Eq. (2), eH2 using Eq. (3).

where $\mathbf{v}$ is a view dependent vector

$\mathbf{v}=\mathbf{K} \frac{\mathbf{R}^{-1} \mathbf{t}}{1+\mathbf{n}_{\pi_{1}}^{\mathrm{T}} / d_{\pi_{1}} \mathbf{R}^{-1} \mathbf{t}}$

and $\mathbf{p}$ is a plane dependent vector. It corresponds with the difference of the two normals and it is the image of the intersection between both planes

$\mathbf{p}=\left(\frac{\mathbf{n}_{\pi_{1}}^{\mathrm{T}}}{d_{\pi_{1}}}-\frac{\mathbf{n}_{\pi_{2}}^{\mathrm{T}}}{d_{\pi_{2}}}\right) \mathbf{K}^{-\mathbf{1}}$.

If we name $\mathbf{v}=\left(v_{1}, v_{2}, v_{3}\right)^{\mathrm{T}}$ and $\mathbf{p}=\left(p_{1}, p_{2}, p_{3}\right)^{\mathrm{T}}$ it turns out that the eigenvalues of the $\mathbf{H}$ homology are $(1,1,1+$ $\left.v_{1} p_{1}+v_{2} p_{2}+v_{3} p_{3}\right)$.

So, the homology has two equal eigenvalues and the third one is related to the motion and to the structure of the scene. These eigenvalues are used to test when two different planes have been computed, and then the epipole and the intersection of the planes can be also computed. The epipole is the eigenvector corresponding to the non-unary eigenvalue and the other two eigenvectors define the intersection line of the planes [5]. In case of small baseline or if there exist only one plane in the scene, epipolar geometry is not defined and only one homography can be computed, so the possible homology $\mathbf{H}$ will be close to identity, up to scale.

In practice, a homology filter is proposed using these ideas. Firstly, we normalize the homology dividing by the median eigenvalue. If there are no two unary eigenvalues, up to a threshold, then the computation is rejected. When the three eigenvalues are similar, we search if the homology is close to identity to avoid the case where two similar homographies explain the scene or the motion. In other case, the homology is accepted.

As said, when two planes are available, the corresponding homographies can be used to obtain the fundamental matrix. The steps carried out to automatically compute the fundamental matrix from lines is summarized in the 

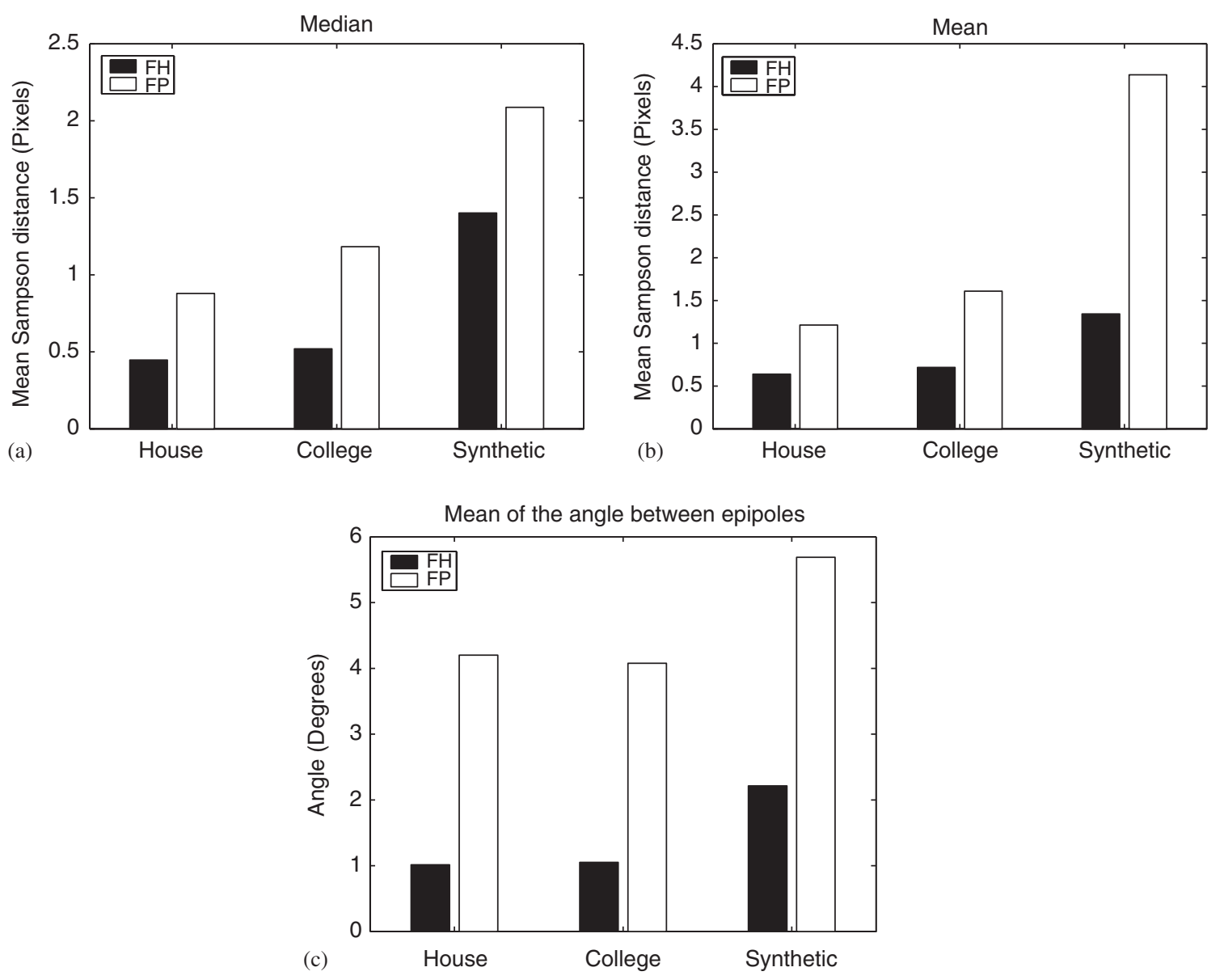

Fig. 6. Mean Sampson distance for 20 test points manually matched in the images of the House, the College and Synthetic. Median (a) and mean (b), in 100 executions. Mean of the angle with the three image pairs, in 100 executions, between the theoretical epipole and the computed one (c). These results are shown for a fundamental matrix computed with homographies (FH), and for a fundamental matrix computed with cross-points (FP).

Algorithm 1.

Algorithm 1 Line matching, homographies and the fundamental matrix

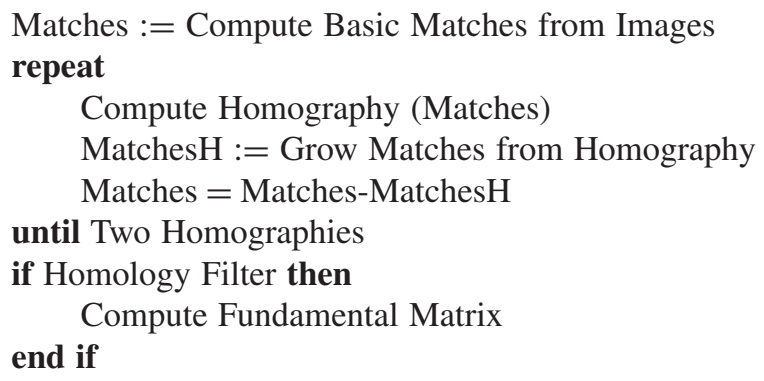

\section{Experimental results}

Many experiments have been carried out with synthetic and real images. The homology filter just commented has been used to determine when a second plane has been ob- tained. In this case the fundamental matrix can be computed and several criteria can be used to measure its accuracy. So, with synthetic images, where the motion and the epipoles are known, we measure the angle between the direction of the computed epipole and the theoretical one. With real images the theoretical epipole is not given and we show the angle of the epipole with respect to the epipole obtained with more than 300 points using the "image-matching" solution [1]. On the other hand, we also measure the first order geometric error computed as the Sampson distance $\left(\mathbf{d}_{S}\right)$ for a set of corresponding test points manually extracted and matched,

$$
\mathbf{d}_{S}=\frac{\left(\mathbf{x}_{2}^{\mathrm{T}} \mathbf{F}_{21} \mathbf{x}_{1}\right)^{2}}{\left(\mathbf{F}_{21} \mathbf{x}_{1}\right)_{f}^{2}+\left(\mathbf{F}_{21} \mathbf{x}_{1}\right)_{s}^{2}+\left(\mathbf{F}_{21}^{\mathrm{T}} \mathbf{x}_{2}\right)_{f}^{2}+\left(\mathbf{F}_{21}^{\mathrm{T}} \mathbf{x}_{2}\right)_{s}^{2}}
$$

being ()$_{f}$ and ()$_{s}$ the first and second components of vectors.

In case of real images, the matches are automatically obtained for the planes in each scene (Figs. 1 and 2). In Table 1 we show the number of matches and the ratio of good matches. In this case, once a homography has been computed, the robust homography computation and the growing matches process has been iteratively repeated twice. The 

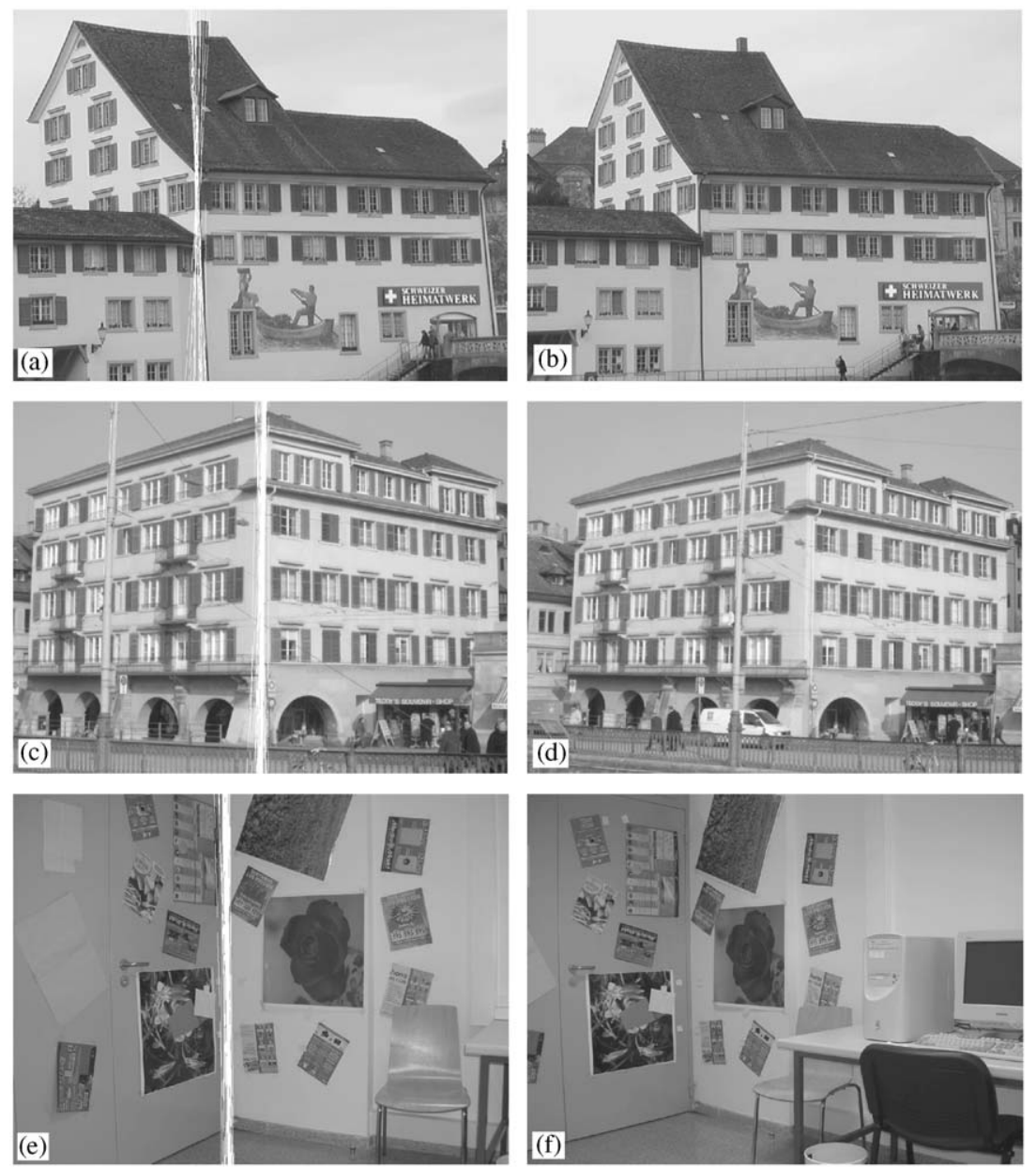

Fig. 7. Intersection lines of two planes through the eigenvalues of the homology. The original images (a)-(d) have been obtained from Ref. [14].
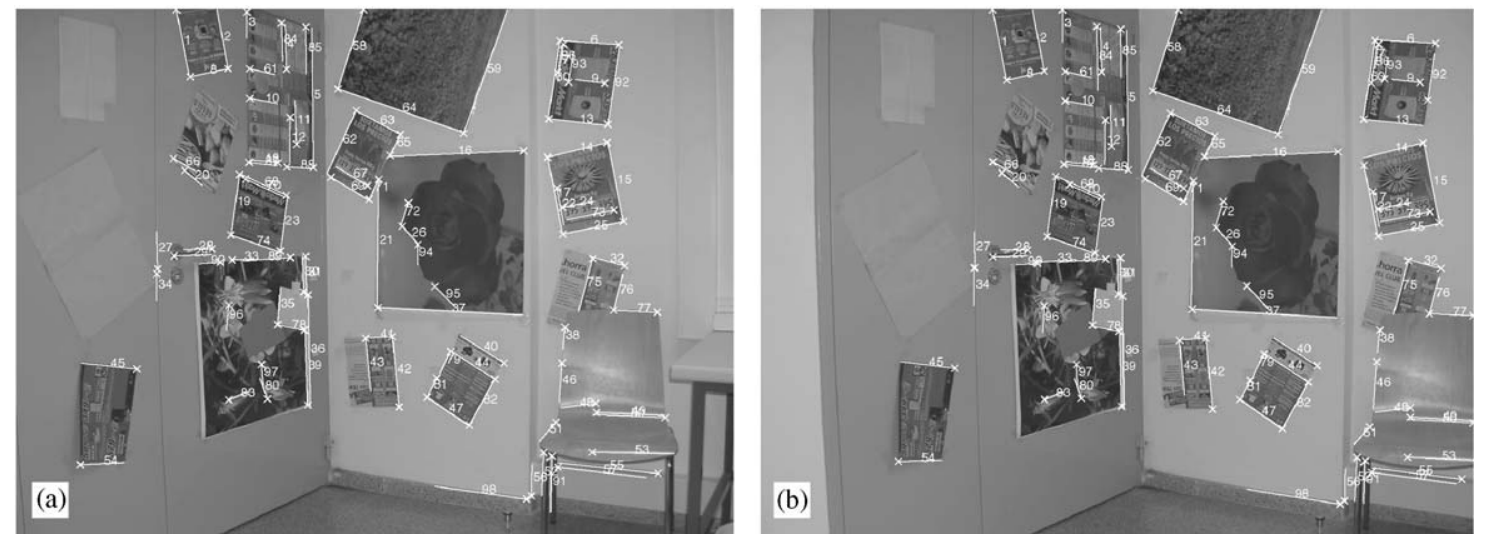

Fig. 8. Computation of matches in image pair of nearly null baseline. The features all over the image are explained with only one homgraphy.

number and quality of final matches can be seen in Figs. 1 and 2.

As we have seen in Section 4 one of the results of the homology is the intersection line of the planes. We have proposed to use a homology filter to avoid situations where a sole homography can be computed or where the homo- graphies do not give a right homology due to noise or bad extraction. In these cases the epipole, the fundamental matrix or the intersection line would be badly computed. In Figs. 3 and 4 we can see the intersection lines for one hundred executions with and without the homology filter for two pairs of test images. As it can be seen the quality of the re- 
sults improves significantly applying the proposed homology filter.

With respect to the fundamental matrix computation, we show the mean of the Sampson distance for 20 test points which are manually matched (Fig. 5(a),(b)). We consider the images of the House, the College, and two Synthetic images. The synthetic images have been created from straight segments in two planes. We have added white noise on the projected tips. This noise has half pixel standard deviation in the segment direction and 10 times less in the perpendicular direction. The experiment has been repeated 100 times and we show mean and median values. In most cases the error is similar to the one obtained with many points which are all over the image (with the fundamental matrix computed with more than 300 points using the "image-matching" software [1], the Sampson distance is about 0.35 pixels). We show the results using Eq. (5), using Eq. (2) and using Eq. (3). The Sampson distance is similar for the three presented ways of computing the fundamental matrix, although it is a bit worse using Eq. (3). Probably the reason is that the first homography is computed more accurate than the second one, because of the iterative method used to compute them. The angle between the epipoles is also shown (Fig. 5(c)). The epipole is a bit better computed using Eq. (2) than using Eq. (5), but the difference is nearly null.

Additionally, we have tested the results when the fundamental matrix is directly computed from the intersection points of the automatically matched lines. We have the matched lines corresponding to each homography. Therefore, they are coplanar and we can get the cross-points between them as corresponding points. We apply the eightpoint algorithm with ransac [5] to compute the fundamental matrix (FP). Then, we compare the mean of the Sampson distance when we use a fundamental matrix computed with the homographies $(\mathrm{FH})$ or with these intersection points of the lines (FP) (Fig. 6(a),(b)). The results with this FP are worse than the ones computed with the homographies. This confirms that the general method to compute the fundamental matrix does not work better than the proposed multiplane method when there are two planes. In addition, the noise is concerning more to these cross-points than to the lines and the homographies, as each of these points accumulate the orientation errors from the two lines used to compute it. For the angle between the epipoles, we find again worse performance using cross-points than using the proposed method with lines and homographies (Fig. 6(c)). Here, it is important to notice that we have used an anisotropic noise model for the tips of the segment (10 times bigger in the line direction than in the perpendicular direction), while an isotropic noise model gives worse results using the cross-points (FP).

We also show results with more images from outdoor and indoor scenes (Fig. 7). Here, we only show the image pair with the intersection lines from 100 runs that passed the homology filter. We can see that the homology filter works even if both planes are not the same size (Fig. 7(a),(b)). What happens in this case is that many times the two found homographies are corresponding to the big plane, but the homology filter discard them properly giving only the fundamental matrix, the epipoles and the intersection line of the planes, as result, when the homology is well conditioned.

In the images of Fig. 8, we show the case where only one homography can be obtained because the baseline is nearly null, what is explained by one homography. So, in 100 iterations the algorithm has not found any correct pair of homographies because the homology filter has detected that in case of computing two homographies they were the same one. At the same time, it can be seen that the number of correct matches is very high (in this case more than 99\%).

\section{Conclusions}

We have presented the computation of epipoles and the intersection of the planes in two views from automatically matched lines. This is carried out through homographies corresponding to planes, which are quite usual in man made environments. The robust computation of matches based on homographies works especially well to automatically eliminate outliers which may appear when there is no information of scene structure or camera motion. The fundamental matrix is properly obtained if the images correspond to motion and scenes which are geometrically well conditioned. If that does not happen, what is detected with the homology filter, a homography is given as result of the algorithm.

Many experiments have been carried out and a summary of the most relevant are presented to show the goodness of the ideas presented. The main contribution of the work is that all is made automatically with only previous tuning of some parameters for image based matching. It is a quite good solution for man made scenes, because in many situations no more than two good planes are available. We are actually working with moment invariants to get a better basic set of matches robust to wide baseline images.

\section{References}

[1] Z. Zhang, R. Deriche, O. Faugeras, Q.T. Luong, A robust technique for matching two uncalibrated images through the recovery of the unknown epipolar geometry, Artif. Intell. 78 (1995) 87-119.

[2] C. Rother, S. Carlsson, Linear multi view reconstruction and camera recovery using a reference plane, Int. J. Comput. Vision 49 (2) (2002) 214-223.

[3] Y. Liu, T.S. Huang, Determining straight line correspondences from intensity images, Pattern Recognition 24 (6) (1991) 489-504.

[4] P. Pritchett, A. Zisserman, Wide baseline stereo matching, in: IEEE Conference on Computer Vision 1998, pp. 754-760.

[5] R. Hartley, A. Zisserman, Multiple View Geometry in Computer Vision, Cambridge University Press, Cambridge, 2000.

[6] J.J. Guerrero, C. Sagüés, Robust line matching and estimate of homographies simultaneously, in: IbPRIA, Pattern Recognition Image Anal., Lecture Notes in Computer Science, vol. 2652, 2003, pp. 297-307.

[7] Q.-T. Luong, O.D. Faugeras, Determining the fundamental matrix with planes: unstability and new algorithms, in: Proceedings of 
the International Conference on Computer Vision and Pattern Recognition, New York, 1993, pp. 489-494.

[8] C. Saguiés, J.J. Guerrero, Visual correction for mobile robot homing, Robotics Autonomous Syst. 50 (1) (2005) 41-49.

[9] M.A. Fischler, R.C. Bolles, Random sample consensus: a paradigm for model fitting with applications to image analysis and automated cartography, Commun. ACM 24 (1981) 381-395.

[10] Q.T. Luong, O. Faugeras, The fundamental matrix: theory, algorithms, and stability analysis, Int. J. Comput. Vision 17 (1) (1996) 43-76.
[11] R.I. Hartley, In defense of the eight-point algorithm, IEEE Trans. on Pattern Anal. and Mach. Intell. 19 (6) (1997) 580-593.

[12] W.H. Press, S.A. Teukolsky, W.T. Vetterling, B.P. Flannery, Numerical Recipes in C: The Art of Scientific Computing, Cambridge University Press, Cambridge, 2002.

[13] L. Zelnik-Manor, M. Irani, Multiview constraints on homographies, IEEE Trans. Pattern Anal. Mach. Intell. 24 (2) (2002) 214-223.

[14] H. Shao, T. Svoboda, L.V. Gool, Zubud-Zurich buildings database for image based recognition. Technical report No. 260 〈http://www.vision.ee.ethz.ch/showroom/zubud//, April 2003.

About the Author-J.J. GUERRERO graduated as Electrical Engineering from the University of Zaragoza in 1989. He obtained his Ph.D. in 1996 from the same institution, and currently he is an Assistant Professor at the Department of Computer Science and Systems Engineering. He gives lectures on control engineering, robotics and 3D computer vision. His research interests are in the area of computer vision, particularly in 3D visual perception, photogrammetry, robotics and vision based navigation.

About the Author-F. ESCUDERO received his M.Sc. Degree in Electrical Engineering from the University of Zaragoza, Spain. His final year project was based upon 3D computer vision, being afterwards granted by the Department of Computer Science and Systems Engineering to continue his research on this topic. Currently he is working as a System Engineer for EDS Spain.

About the Author-A.C. MURILLO received her M.Sc. Degree in Informatics Engineering in the University of Zaragoza in 2003. Currently she is a Ph.D. student at the Department of Computer Science and Systems Engineering in the same university. Her research interests are in the area of computer vision, focusing in multi-view geometry, wide-baseline matching and mobile robotics applications.

About the Author-C. SAGÜÉS received his M.Sc. and Ph.D. degrees from the University of Zaragoza, Spain. During the course of his Ph.D. he was working on force and infrared sensors for robots. Since 1994, he has been with the Department of Computer Science and Systems Engineering as an Assistant Professor and currently he is the Head Teacher. He gives lectures on control engineering, robotics for B.Sc. students and he also gives lectures on 3D computer vision for M.Sc. students. His current research interests include computer vision, visual robot navigation and visual servoing being the author of several conference and journal papers related to these topics. 